\title{
Co-evolution of tumor-associated macrophages and tumor neo-vessels during cervical cancer invasion
}

\author{
SHUTING JIANG ${ }^{1 *}$, YUEHONG YANG ${ }^{2 *}$, MIN FANG $^{3 *}$, XIANGLANG LI ${ }^{4}$, \\ XIUXUE YUAN $^{5}$ and JINGPING YUAN ${ }^{6}$ \\ ${ }^{1}$ Department of Breast Surgery, Changzhou Maternal and Child Health Care Hospital, Changzhou, Jiangsu 213003; \\ ${ }^{2}$ Department of Pathology, Wuhan Center Hospital, Wuhan, Hubei 430014; \\ ${ }^{3}$ Department of Radiation Oncology, Zhejiang Cancer Hospital, Hangzhou, Zhejiang 310022; \\ ${ }^{4}$ Department of Oncology, Hunan Aerospace Hospital, Changsha, Hunan 410205; \\ ${ }^{5}$ Medical College of Wuhan University of Science and Technology, Wuhan, Hubei 430065; \\ ${ }^{6}$ Department of Pathology, Renmin Hospital of Wuhan University, Wuhan, Hubei 430060, P.R. China
}

Received May 14, 2015; Accepted July 20, 2016

DOI: $10.3892 / \mathrm{ol} .2016 .5014$

\begin{abstract}
Considering the crucial significance of the tumor microenvironment in cancer development and progression, the present study aimed to investigate the changes in macrophages and angiogenesis during the cervical cancer (CC) progression process from chronic cervicitis to cervical intraepithelial neoplasia grades I-III (CIN I-III) to CC. This investigation included quantitative analysis and assessment of the spatial associations between tumor-associated macrophages (TAMs) and tumor neo-vessels. The conventional immunohistochemistry staining technique was used to detect cluster of differentiation (CD)68 and CD105 biomarker expression for TAMs and tumor neo-vessels, respectively. In addition, with the assistance of quantum dot (QD)-based two-component in situ imaging technology, the expression of the TAMs and tumor neo-vessels could be observed simultaneously. The quantitative analysis and co-evolution of the TAMs and tumor neo-vessels could then be processed. During the progression process from chronic cervicitis to cervical CIN I-III, and ultimately to invasive $\mathrm{CC}$, the expression of the macrophages and neo-vessels in the tumor microenvironment increased
\end{abstract}

Correspondence to: Professor Xiuxue Yuan, Medical College of Wuhan University of Science and Technology, 947 Heping Road, Wuhan, Hubei 430065, P.R. China

E-mail: exiu2001@sohu.com

Professor Jing ping Yuan, Department of Pathology, Renmin Hospital of Wuhan University, 238 Jiefang Road, Wuhan, Hubei 430060, P.R. China

E-mail: yuanjingping2003@aliyun.com

*Contributed equally

Key words: cervival cancer, tumor-associated macrophages, tumor neo-vessels, quantum dots synchronously. According to the quantitative analysis results, the median value of the TAM density was higher in the CC group $(5,540.14)$ than in the CIN I-III group $(2,502.17)$ and the chronic cervicitis group $(1,403.31)$, with statistical significance in all three groups ( $\mathrm{P}<0.001$, for between-group comparisons). The number of neo-vessels was also much higher in the CC group $(n=27)$ than in the CIN I-III group $(n=17)$ or the chronic cervicitis group $(n=6.5)$, with statistical significance in all three groups ( $\mathrm{P}<0.001$, for between-group comparisons). These findings demonstrated the great significance and close association of TAMs and tumor angiogenesis during $\mathrm{CC}$ development and progression. Thus, QDs-based in situ and simultaneous imaging of key cancer molecules may provide insights with regard to the biology of cancer invasion.

\section{Introduction}

Cervical cancer (CC) is the third most common cancer and the fourth leading cause of cancer-associated mortality in females worldwide (1). CC is the leading cause of cancer-associated mortality in young women, and the most common cause of cancer-associated mortality among women in the developing world (2). For a long period of time, focus has been placed on cancer cells, and the importance of the tumor microenvironment has not been recognized. However, it is now known that cancer is not merely a local disease, but a systematic disease, involving a group of rogue cells (3), in which the tumor microenvironment plays a pivotal role.

The tumor microenvironment is a complex community (3), including cancer cells, stromal tissue with immune cells, fibroblasts, myofibroblasts, cytokines and vascular tissue, as well as the extracellular matrix surrounding these components (4). In the 1880 s, Paget reported the 'seed and soil' theory (5), which indicated that in order to fully comprehend cancer initiation and progression, a focus must not only be placed on the cancer cells, 'the seed', but also on 'the soil' from which nutrients, oxygen and signals for growth and development are derived, and within which macrophages and 
tumor angiogenesis (6), the most representative factors in the tumor microenvironment, play crucial roles in the development of tumors.

Macrophages are important representatives of immune function during cancer progression process (7), acting as vital components of the host's defense, and as antigen-presenting cells and effector cells $(8,9)$. Macrophages are derived nearly completely from circulating monocytes, and acquire unique phenotypic characteristics with diverse functions in response to environmental signals (10). As human solid tumors can often be anatomically classified into the areas of a cancer nest, the invading edge and the peritumoral stroma, each with distinct compositions and functional properties $(11,12)$, the tumor-associated monocytes/macrophages (TAMs) exhibit different phenotypes and functions at these distinct tumor sites (13-15). Macrophages in normal or inflamed tissues exhibit spontaneous anti-tumor activity, whereas TAMs could be oriented towards promoting tumor growth, remodeling tissues, promoting angiogenesis and suppressing adaptive immunity (16-18). Tumor angiogenesis, another key component in the tumor microenvironment, also plays an essential role during cancer progression (19). In 1971, Folkman first proposed the vital role of angiogenesis in the growth of tumors, describing tumors as 'hot and bloody' (20). Angiogenesis occurs in the normal and vital processes of growth and development, as well as in wound healing and in granulation tissue formation (20). However, angiogenesis is also essential in the tumor transition between benign and malignant states, resulting in the use of angiogenesis inhibitors to treat cancer (21).

In addition, studies have shown that TAMs could promote tumor angiogenesis and metastasis (22). TAMs and cancer cells can secrete various growth factors, angiogenic factors and a variety of enzymes, which play important roles in angiogenesis (23). Thus, TAMs and tumor angiogenesis jointly promote cancer invasion and metastasis. Therefore, it is urgent to develop a technique, which can image multiple components in situ so as to study the co-evolution of TAMs and tumor angiogenesis during cancer invasion. Quantum dots (QDs) are a novel class of semiconductor nanocrystals of $1-10 \mathrm{~nm}$ in diameter $(24,25)$, which have unique optical and electrical properties, including narrow and symmetric emission spectra of light, good light stability, strong fluorescence intensity, a long fluorescence schedule of 30-100 nsec and a changeable emission wavelength with the QD size, and are thus of great potential in the fields of biological imaging and molecular markers $(26,27)$. Major advances have emerged as a result of using QDs for highly sensitive cellular imaging over the past decade (28). In comparison with organic dyes and fluorescent proteins, QDs exhibit the unique features of size and composition tunable light emission, enhanced signal brightness and resistance to photobleaching (29). In this study, the special properties of QDs were harnessed to better understand the role of tumor microenvironment during cancer progression, which was also reported in our previous study (30).

Therefore, the present study aimed to observe the spatial and temporal distributions of TAMs and tumor angiogenesis during the progression from chronic cervicitis to cancer through the use of conventional IHC and QD-based two-component in situ imaging technology, in order to investigate the co-evolution between the aforementioned components of the tumor microenvironment and CC cells.

\section{Materials and methods}

Samples. A total of 90 paraffin-embedded cervical samples were collected from Wuhan Municipality Central Hospital (Wuhan, Hubei, China) between January 2013 and December 2014, including 30 cases of chronic cervicitis, 30 CIN I-III biopsy samples and $30 \mathrm{CC}$ resected samples. The study protocol was approved by the Ethics Committee of Wuhan Municipality Central Hospital. Written informed consent was obtained from all patients prior to surgery, with permission to use the specimens for scientific research purposes and clinicopathological studies.

Reagents. Mouse anti-human monoclonal antibody against macrophage (catalog no. MA1-38069; dilution, 1/100; ABR Affinity BioReagents, Golden, CO, USA) and rabbit anti-human monoclonal antibody against CD105 (catalog no. ab169545; dilution, 1/300; Abcam, Cambridge, MA, USA) were used. The QDs were goat anti-mouse secondary antibody QD-525 (dilution, 1/100; Invitrogen; Thermo Fisher Scientific Inc., Waltham, MA, USA) and goat anti-rabbit QD-585 (dilution, 1/200; Invitrogen; Thermo Fisher Scientific Inc.), and the remaining conventional reagents were purchased from Dako (Glostrup, Denmark).

Immunohistochemistry. The use of IHC followed the instructions of our previously established technical procedure (31). Briefly, tissue sections ( $4-\mu \mathrm{m}$ thick) were treated by de-paraffinizing, hydration, antigen retrieval and washing in deionized water prior to proceeding to the following imaging studies. The main steps of the IHC were as follows: The endogenous peroxidase activity was blocked by immersion in $3 \% \mathrm{H}_{2} \mathrm{O}_{2}$ for $10 \mathrm{~min}$ to prevent any non-specific binding. After being repaired with pH 6.0 citric acid under high pressure, the slides were first incubated with primary antibody for $30 \mathrm{~min}$, then with the corresponding secondary antibody for $30 \mathrm{~min}$. Next, they were colored with 3,3'-diaminobenzidine for $10 \mathrm{~min}$, stained with hematoxylin, dehydrated to transparency and covered with neutral gum. Phosphate-buffered saline (PBS) was used instead of a primary antibody for the negative control.

QD-based molecular imaging. For QD-based immune-fluorescent imaging, subsequent to being blocked with $2 \%$ bovine serum antigen (BSA) for $30 \mathrm{~min}$ at $37^{\circ} \mathrm{C}$, the sections were incubated with the aforementioned primary antibodies at $37^{\circ} \mathrm{C}$ for $2 \mathrm{~h}$. Once washed with PBS 3 times, the sections were incubated with the corresponding secondary antibodies of the QDs for $2 \mathrm{~h}$ at $37^{\circ} \mathrm{C}$ after another blockade with $2 \%$ BSA for $30 \mathrm{~min}$. For the in situ two-component imaging, the primary antibodies, including mouse anti-human monoclonal antibody against macrophage (catalog no. MA1-38069; dilution, 1/100) and rabbit anti-human monoclonal antibody against CD105 (catalog no. ab169545; dilution, 1/300) were mixed. The sections were incubated with corresponding mixed secondary antibodies 

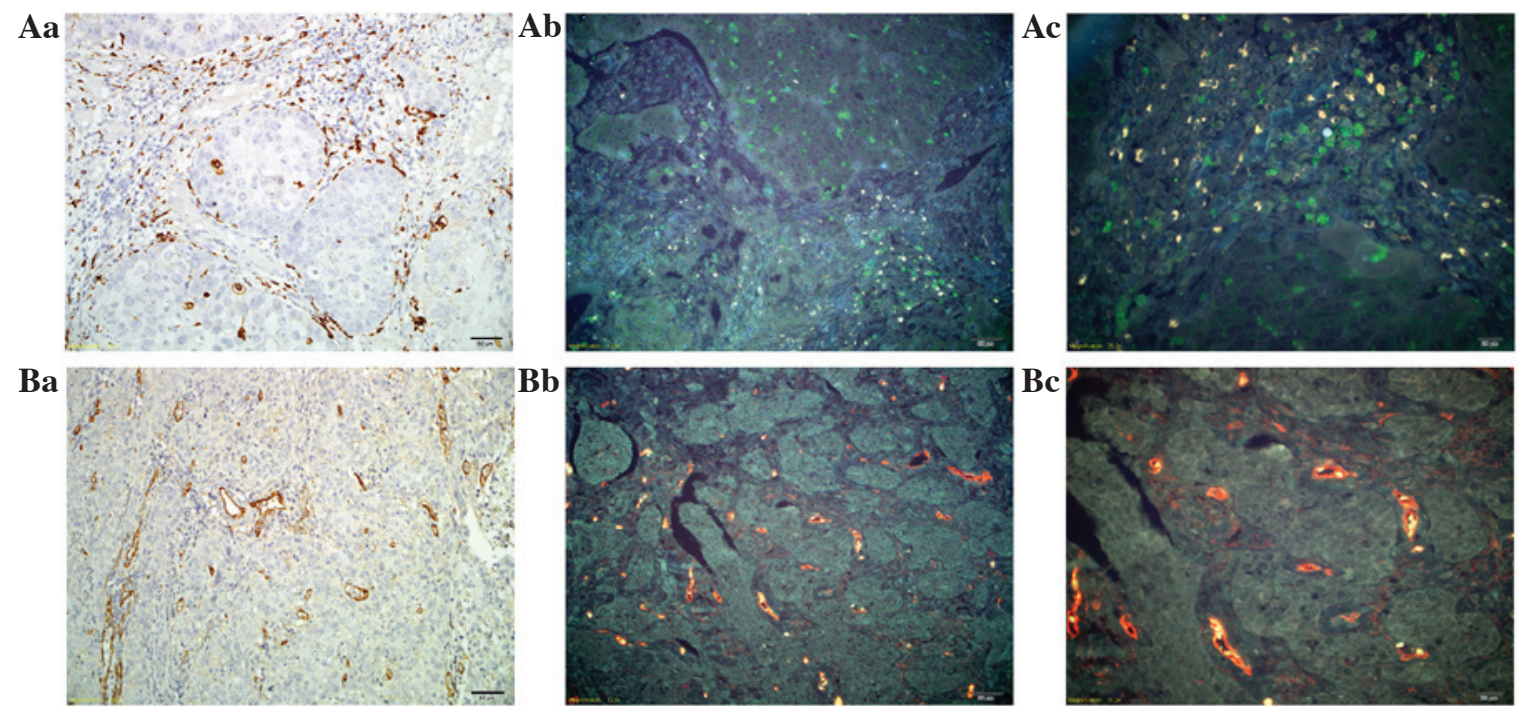

Figure 1. Establishment of QD-based molecular imaging of cervical cancer tissue. (Aa) Staining of TAMs by IHC at x200 magnification, and the expression of TAMs marked by CD68 with QD-525 (green color) at (Ab) x200 and (Ac) x400 magnification. (Ba) Staining of neo-vessels by IHC at x200 magnification, and the expression of tumor neo-vessels marked by CD105 with QD-585 (yellow color) at (Bb) x200 and (Bc) x400 magnification. TAMs, tumor-associated macrophages; IHC, immunohistochemistry; QD, quantum dot; CD, cluster of differentiation.

of QD-525 and QD-585. The sections were assessed after washing.

Image acquisition and processing. All sections were observed under an Olympus BX51 fluorescence microscope (Olympus, Tokyo, Japan). Images of routine IHC and QDs were collected under bright field and obtained by ultraviolet (UV) excitation (UV, 330-385 nm), respectively, and all images were obtained using a CRi Nuance multispectral imaging system (Cambridge Research and Instrumentation, Inc., Woburn, MA, USA). Each tissue section was observed by two double-blind randomized observations, first at a low magnification (x100) in regions with the highest density of TAMs and tumor neo-vessels ('hot spots'), and then at a high magnification (x200) as capture images in three selected hot spots. After using the aforementioned spectral separation system, the density of the TAMs and the tumor neo-vessel number were calculated, and the mean values were recorded for the cases.

Statistical analysis. The Mann-Whitney U test or the Kruskal-Wallis H test was analyzed using the SPSS 19.0 statistical software (IBM SPSS, Armonk, NY, USA), and two-tailed $\mathrm{P}<0.05$ was considered to indicate a statistically significant difference.

\section{Results}

Establishment of $Q D$-based imaging of $C C$. IHC is a significant technology for clinical application, which utilizes the basic principles of immunology and the antigen-antibody reaction (32). The present study first examined the expression of TAMs and tumor neo-vessels using traditional IHC in CC tissue. Macrophages marked by CD68 were stained brown on the cell membrane and in the cytoplasm (Fig. 1Aa). Tumor neo-vessels marked by CD105 were stained brown in tumor stroma, and were mainly located around the cancer nests (Fig. 1Ba). Then, we used QDs-based probes to image TAMs (Fig. 1 $\mathrm{Ab}$ and $\mathrm{Ac}$ ) and tumor neo-vessels (Fig. 1Bb and Bc). The QDs fluorescence specifically labeled TAMs with green color and tumor neo-vessels with yellow color, without non-specific binding. The background was tissue auto-fluorescence, which did not interfere with the targets, but could assist in identifying tissue morphology as a contrasting background. Compared with the results of the IHC, the success of QD-based imaging was confirmed, as the same regions of the tissue sections were marked.

Dynamic changes in the tumor microenvironment during CC progression. The imaging of multiple molecular markers in tumor and peritumoral tissues in situ can demonstrate the key events of cancer invasion. Focus is placed on two major components of cancer invasion: Monocyte infiltration represented by macrophages (CD68) and tumor vascularization represented by CD105 (30). From normal tissue to invasive CC tissue, the tumor microenvironment was presented as significantly heterogeneous in terms of TAMs and tumor neo-vessels when assessed with QD-based nanoprobes. In normal tissue, there were few macrophages under the basement membrane (BM), and few regular and intact vessels with an intact and linear BM (Fig. 2Aa-Ac). In CC in situ tissue, the cancer cells broke through the BM to form a micro-invasive front; the TAMs presented mainly at the invasive front in the stroma together with irregular and incomplete tumor neo-vessels (Fig. 2Ba-Bc). In well-differentiated invasive CC, the TAMs were extensively distributed among the cancer cells and in the stroma, with prominent tumor neo-vessels surrounding tumor nests (Fig. 2Ca-Cc). Compared with the well-differentiated invasive $\mathrm{CC}$, in the poorly-differentiated invasive $\mathrm{CC}$, the TAMs were abundant with a clustered morphology. Meanwhile, tumor neo-vessels were greater in number compared with in the well-differentiated invasive $\mathrm{CC}$ and were distributed in 

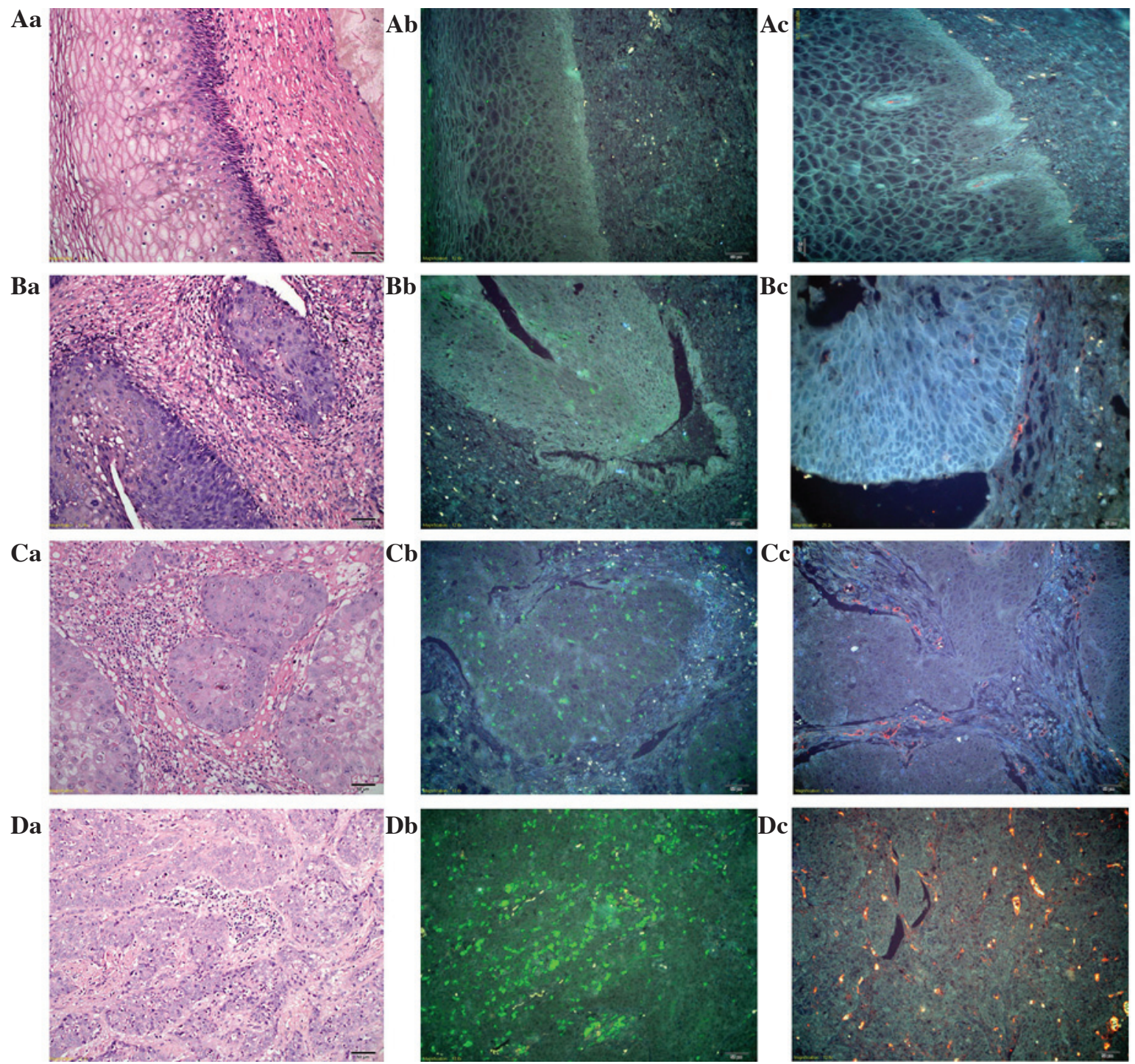

Figure 2. Dynamic changes in the tumor microenvironment during CC progression. (Aa) H\&E staining, (Ab) TAM staining with QDs-525 and (Ac) tumor neo-vessel staining with QD-585, in normal tissue. (Ba) H\&E staining, (Bb) TAM staining with QDs-525 and (Bc) tumor neo-vessel staining with QD-585, in CC in situ tissue. (Ca) H\&E staining, (Cb) TAM staining with QD-525 and (Cc) tumor neo-vessel staining with QD-585, in well-differentiated CC. (Da) H\&E staining, (Db) TAM staining with QD-525 and (Dc) tumor neo-vessel staining with QD-585, in poorly-differentiated CC. Aa-Ac, Ba and Bb, Ca-Cc and Da-Dc: Magnification, x200; and Bc: Magnfication, x400. TAMs, tumor-associated macrophages; QD, quantum dot; CC, cervical cancer; H\&E, hematoxylin and eosin.

a scattered manner with an irregular diameter and generally small morphology (Fig. 2Da-Dc). Overall, during the CC progression process from normal tissue to invasive $\mathrm{CC}$, it could be clearly observed that the TAMs (green color) increased gradually in density in Fig. $2 \mathrm{Ab} \rightarrow \mathrm{Bb} \rightarrow \mathrm{Cb} \rightarrow \mathrm{Db}$, as did the tumor neo-vessels (yellow color) in Fig. $2 \mathrm{Ac} \rightarrow \mathrm{Bc} \rightarrow \mathrm{Cc} \rightarrow \mathrm{Dc}$.

QD-based double staining of TAMs and tumor neo-vessels in situ. With the advantages of the multiplexed imaging of QDs, TAMs and tumor neo-vessels were simultaneously visualized in the tumor tissues. This method breaks the traditional technological barriers to acquire multiple bio-information in situ and made it possible to analyze the temporal-spatial process and morphological characteristics of cancer invasion. In chronic cervicitis tissue, TAMs were barely expressed and few vessels were distributed at the BM with regular and conventional morphology (Fig. 3Aa-Ac). In CC in situ tissues, the density of the TAMs increased and they were distributed in close vicinity to tumor neo-vessels or were found lodging in vessels (Fig. 3Ba-Bc). In invasive CC, the TAMs were scattered all around the tumor nests and the stroma, with cluster aggregation, accompanied with rich tumor angiogenesis (Fig. 3Ca-Cc). Based on the simultaneous double staining of TAMs and tumor neo-vessels in situ, it was clearly noted that the TAMs and tumor angiogenesis increased simultaneously and were distributed in close proximity during $\mathrm{CC}$ progression.

Quantitative analysis of TAMs and tumor neo-vessels. A set of 90 patients with the three most common pathological types of cervical disease, namely chronic cervicitis, CIN I-III and $\mathrm{CC}$, were selected, with 30 cases in each group. The tissue slides from each patient were quantitatively analyzed for TAM density and tumor neo-vessel number. According to the quantitative analysis results, the median value of TAM density was higher in the CC group $(5,540.14)$ than in the CIN I-III 

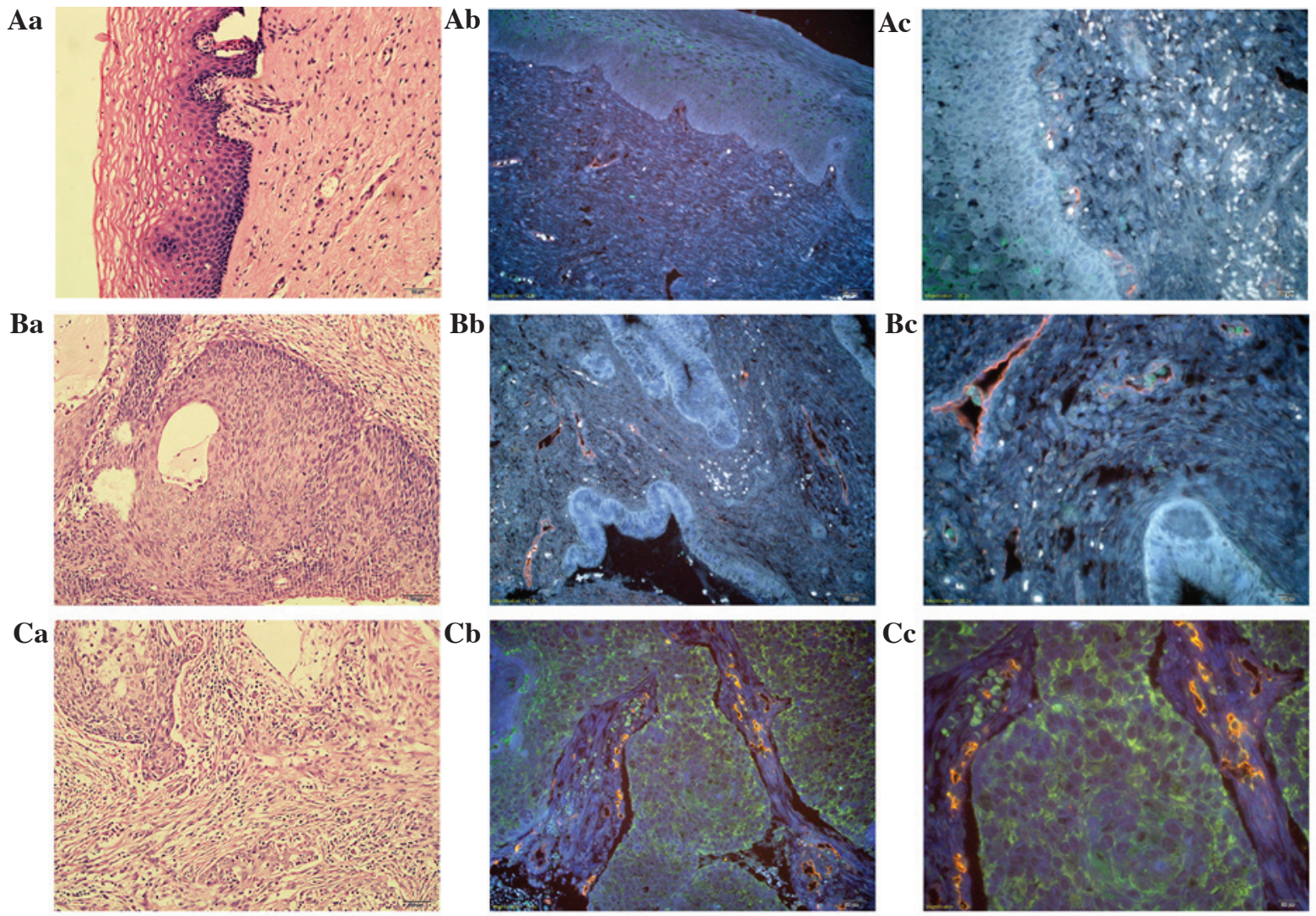

Figure 3. QD-based TAM and tumor neo-vessel staining in situ simultaneously. H\&E staining of (Aa) chronic cervicitis tissue, (Ba) CC in situ and (Ca) invasive CC tissue at x200 magnification, and TAMs and tumor neo-vessels simultaneously stained with QD-525 and QD-585 in chronic cervicitis tissue at (Ab) x200 and (Ac) x400 magnification, in CC in situ at (Bb) x200 and (Bc) x400 magnification, and in invasive $\mathrm{CC}$ tissue at (Cb) x200 and (Cc) x400 magnification. TAMs, tumor-associated macrophages; QD, quantum dot; CC, cervical cancer; H\&E, hematoxylin and eosin.

group $(2,502.17)$ and the chronic cervicitis group $(1,403.31)$, with statistical significance in all three groups $(\mathrm{P}<0.001$, for between-group comparisons). The number of neo-vessels was also much higher in the CC group $(n=27)$ than in the CIN I-III group ( $\mathrm{n}=17)$ or the chronic cervicitis group $(\mathrm{n}=6.5)$, with statistical significance in all three groups $(\mathrm{P}<0.001$, for between-group comparisons). In addition, during the progression process from chronic cervicitis to CIN I-III to CC, the TAM density and the number of tumor neo-vessels gradually increased and were distributed in close proximity.

\section{Discussion}

Tumor invasion and metastasis is a multi-factor, multi-stage, gradually developing and complex dynamic process $(33,34)$, involving not only the abnormal expression of a variety of genes of the cancer cells themselves and the activation of multiple signaling pathways, but also the interaction between tumor cells and the surrounding microenvironment (35). The components of the tumor microenvironment are numerous, and are composed of stromal cells and interstitial cells, including fibroblasts, endothelial cells, infiltrating immune cells and structural support collagen (36). Tumor inflammation and tumor angiogenesis are considered indispensible for cancer invasion. The present study indicated that the tumor microenvironment plays a significant role in cancer progression, involving immune cell infiltration and tumor angiogenesis.
Endothelial cells and immunocytes in the microenvironment around the tumor cells can promote the progression of cancer $(4,37)$. The infiltration of macrophages distributed in the surrounding tumor tissues is the most important ingredients of immune infiltration research (38). Therefore, they are also known as 'tumor-associated macrophages', infiltrating the peripheral vasculature or intravasculature, which are important contact media between inflammation and cancer (39). In addition, the process of tumor angiogenesis is another crucial hallmark of cancer progression. Tumor tissue must be supplied with nutrients from the blood vessels in order to grow (40). Typically, tumor neovessels are disordered with high permeability, which results in hypoperfusion. Therefore, tumor angiogenesis is usually incomplete and of inferior maturity, which could provide strong conditions for tumor invasion and metastasis (41). Recent studies have showed that macrophages express a series of factors to promote tumor progression through the occurrence of hypoxia, angiogenesis, immune suppression and metastasis (42). It was shown that the macrophages infiltrated into tumor tissues and adjacent tissues, mainly in the junctions between cancer nests and interstitial cells, particularly in the places where the blood vessels were rich, which is consistent with the present findings. During the CC invasion progress, the TAMs and tumor neo-vessels simultaneously increased surrounding the cancer nests and were located in close proximity. Macrophages can also secrete vascular endothelial growth factor and other factors 
to promote the formation of vascular endothelial cells (43) and the sprouting of blood vessels through structural support, so as to increase the tumor vascular network (44). Thus, the combinational analysis of TAMs and tumor neo-vessels could better reveal the cancer invasion biology and guide clinical strategy.

However, current methods for tumor invasion and metastasis research, including polymerase chain reaction, western blotting and IHC, could be used to perform qualitative and semi-quantitative research. However, these methods cannot show the spatial distribution of the key components during the development in situ within cancer cells $(45,46)$. In addition, given the complex compositions and functions of the tumor microenvironment, the single-molecule research techniques, such as conventional IHC, are difficult to use to accurately reflect the interactions between cancer cells and the tumor microenvironment. While one component such as the TAMs could be observed directly at one time, there must be another imaging procedure to produce the image for tumor neo-vessels. The shortcoming of these conventional techniques is that subtle essential information on the major components of the tumor microenvironment cannot be obtained simultaneously, thus leading to information loss and low resolution of different components in situ (34). Therefore, in order to solve this problem, there is an urgent requirement to develop multi-situ molecular imaging techniques so as to better guide the clinical treatment.

QD-based molecular imaging and multispectral analysis could make a unique contribution in this regard, as the current study demonstrated. QDs are engineered nanoparticles with unique optical properties and have shown great potential for biomedical application. Compared with organic dyes and fluorescent proteins, due to its unique size and surface features, QDs have many advantages such as enhanced fluorescence brightness and strong resistance to photobleaching. In addition, different QDs can be excited simultaneously by a single light source, which provides significant advantages for the multiplexed detection of targets, suitable for investigating the several key components in tumor microenvironment (27). In the present study, with the aid of QD-based in situ two-component imaging of the tumor microenvironment, the TAM and CD105 levels gradually and significantly increased after quantitative analysis, and the TAMs were found to be scattered all around the tumor nests and the stroma with cluster aggregation, accompanied with rich tumor angiogenesis during the progression process from chronic cervicitis and CIN I-III to invasive CC. Therefore, instead of producing artificial overlay images by conventional imaging techniques, this QD-based molecular imaging technique could provide authentic multicolor images to better reveal the complex tumor microenvironment in cancer tissues.

In conclusion, the present study combined the advantages of QD-based multiplexed imaging in situ to detect macrophages and tumor angiogenesis in chronic cervicitis, cervical CIN I-III and CC tissues, emphasizing the importance of the overall analysis of the tumor microenvironment during cancer invasion and metastasis, and enhancing the understanding of the process of tumor invasion. The findings demonstrated that the TAMs and tumor neo-vessels together promoted cancer invasion. It was also suggested that a simple one-component study based on cancer cells or the tumor microenvironment is insufficient to fully reflect the cancer invasion process. Therefore, there is an urgent requirement for the development of in situ multi-component imaging technology and an image analysis method.

\section{Acknowledgements}

The authors would like to acknowledge the financial support provided by the Natural Science Foundation of Hubei Province (grant no. 2013CFB374).

\section{References}

1. Siegel R, Naishadham D and Jernal A: Cancer statistics, 2013. CA Cancer J Clin 63: 11-30, 2013.

2. Limmer K, LoBiondo-Wood G and Dains J: Predictors of cervical cancer screening adherence in the United States: A systematic review. J Adv Pract Oncol 5: 31-41, 2014.

3. Bizzarri M and Cucina A: Tumor and the microenvironment: A Chance to reframe the paradigm of carcinogenesis? Biomed Res Int 2014: 934038, 2014.

4. Polyak K, Haviv I and Campbell IG: Co-evolution of tumor cells and their microenvironment. Trends Genet 25: 30-38, 2009

5. Paget S: The distribution of secondary growths in cancer of the breast. Lancet 133: 571-573, 1889.

6. Sun T, Yang Y, Luo X, Cheng Y, Zhang M, Wang K and Ge C: Inhibition of tumor angiogenesis by interferon- $\gamma$ by suppression of tumor-associated macrophage differentiation. Oncol Res 21: 227-235, 2014.

7. Liu Q, Zhang A, Xu W and Dong J: A new view of the roles of blood flow dynamics and Kupffer cell in intra-hepatic metastasis of hepatocellular carcinoma. Med Hypotheses 77: 87-90, 2011.

8. Lewis CE and Pollard JW: Distinct role of macrophages in different tumor microenvironments. Cancer Res 66: 605-612, 2006.

9. Gordon S and Taylor PR: Monocyte and macrophage heterogeneity. Nat Rev Immunol 5: 953-964, 2005.

10. Biswas SK and Mantovani A: Macrophage plasticity and interaction with lymphocyte subsets: Cancer as a paradigm. Nat Immunol 11: 889-896, 2010.

11. Budhu A, Forgues M, Ye QH, Jia HL, He P, Zanetti KA, Kammula US, Chen Y, Qin LX, Tang ZY and Wang XW: Prediction of venous metastases, recurrence, and prognosis in hepatocellular carcinoma based on a unique immune response signature of the liver microenvironment. Cancer Cell 10: 99-111, 2006.

12. Vakkila J and Lotze MT: Inflammation and necrosis promote tumour growth. Nat Rev Immunol 4: 641-648, 2004.

13. Qian BZ and Pollard JW: Macrophage diversity enhances tumor progression and metastasis. Cell 141: 39-51, 2010.

14. Mantovani A, Sozzani S, Locati M, Allavena P and Sica A: Macrophage polarization: Tumor-associated macrophages as a paradigm for polarized M2 mononuclear phagocytes. Trends Immunol 23: 549-555, 2002.

15. Gao B, Jeong WI and Tian Z: Liver: An organ with predominant innate immunity. Hepatology 47: 729-736, 2008.

16. Qian BZ and Pollard JW: New tricks for metastasis-associated macrophages. Breast Cancer Res 14: 316, 2012.

17. Kuang DM, Zhao Q, Peng C, Xu J, Zhang JP, Wu C and Zheng L: Activated monocytes in peritumoral stroma of hepatocellular carcinoma foster immune privilege and disease progression through PD-L1. J Exp Med 206: 1327-1337, 2009.

18. Zhang JP, Yan J, Xu J, Pang XH, Chen MS, Li L, Wu C, Li SP and Zheng L: Increased intratumoral IL-17-producing cells correlate with poor survival in hepatocellular carcinoma patients. J Hepatol 50: 980-989, 2009.

19. Yu D, Zhuang L, Sun X, Chen J, Yao Y, Meng K and Ding Y: Particular distribution and expression pattern of endoglin (CD105) in the liver of patients with hepatocellular carcinoma. BMC Cancer 7: 122, 2007.

20. Folkman J: Tumor angiogenesis: Therapeutic implications. N Engl J Med 285: 1182-1186, 1971.

21. Devy L, Huang L, Naa L, Yanamandra N, Pieters H, Frans N, Chang E, Tao Q, Vanhove M, Lejeune A, et al: Selective inhibition of matrix metalloproteinase-14 blocks tumor growth, invasion, and angiogenesis. Cancer Res 69: 1517-1526, 2009. 
22. Guzmán-Medrano R, Arreola-Rosales RL, Shibayama M, Silva-Olivares DA, Bologna-Molina R and Rodríguez MA: Tumor-associated macrophages and angiogenesis: A statistical correlation that could reflect a critical relationship in ameloblastoma. Pathol Res Pract 208: 672-676, 2012.

23. Zhu JY, Sun QK, Wang W and Jia WD: High-level expression of HOXB13 is closely associated with tumor angiogenesis and poor prognosis of hepatocellular carcinoma. Int J Clin Exp Pathol 7: 2925-2933, 2014

24. Resch-Genger U, Grabolle M, Cavaliere-Jaricot S, Nitschke R and Nann T: Quantum dots versus organic dyes as fluorescent labels. Nat Methods 5: 763-775, 2008.

25. Madani SY, Shabani F, Dwek MV and Seifalian AM: Conjugation of quantum dots on carbon nanotubes for medical diagnosis and treatment. Int J Nanomedicine 8: 941-950, 2013.

26. Chen C, Peng J, Sun SR, Peng CW, Li Y and Pang DW: Tapping the potential of quantum dots for personalized oncology: Current status and future perspectives. Nanomedicine (Lond) 7: 411-428, 2012.

27. Fang M, Peng CW, Pang DW and Li Y: Quantum dots for cancer research: Current status, remaining issues, and future perspectives. Cancer Biol Med 9: 151-163, 2012.

28. Hoshino A, Manabe N, Fujioka K, Suzuki K, Yasuhara M and Yamamoto K: Use of fluorescent quantum dot bioconjugates for cellular imaging of immune cells, cell organelle labeling, and nanomedicine: Surface modification regulates biological function, including cytotoxicity. J Artif Organs 10: 149-157, 2007.

29. Chen LD, Liu J, Yu XF, He M, Pei XF, Tang ZY, Wang QQ, Pang DW and Li Y: The biocompatibility of quantum dot probes used for the targeted imaging of hepatocellular carcinoma metastasis. Biomaterials 29: 4170-4176, 2008.

30. Fang M, Peng CW, Yuan JP, Zhang ZL, Pang DW and Li Y: Coevolution of the tumor microenvironment revealed by quantum dot-based multiplexed imaging of hepatocellular carcinoma. Future Oncol 9: 1029-1037, 2013

31. Chen C, Peng J, Xia HS, Yang GF, Wu QS, Chen LD, Zeng LB, Zhang ZL, Pang DW and Li Y: Quantum dots-based immunofluorescence technology for the quantitative determination of HER2 expression in breast cancer. Biomaterials 30: 2912-2918, 2009.

32. Peuscher A, Gassler N, Schneider U, Thom P, Rasche S, Spiegel H and Schillberg S: An immunohistochemical assay on human tissue using a human primary antibody. J Immunoassay Immunochem 35 : 322-334, 2014

33. Sulzmaier FJ and Ramos JW: RSK isoforms in cancer cell invasion and metastasis. Cancer Res 73: 6099-6105, 2013.

34. Fang M, Yuan JP, Peng CW, Pang DW and Li Y: Quantum dots-based in situ molecular imaging of dynamic changes of collagen IV during cancer invasion. Biomaterials 34: 8708-8717, 2013.
35. Friedl P and Alexander S: Cancer invasion and the microenvironment: Plasticity and reciprocity. Cell 147: 992-1009, 2011.

36. Nguyen-Ngoc KV, Cheung KJ, Brenot A, Shamir ER, Gray RS Hines WC, Yaswen P, Werb Z and Ewald AJ: ECM microenvironment regulates collective migration and local dissemination in normal and malignant mammary epithelium. Proc Nat Acad Sci USA 109: E2595-E2604, 2012.

37. Zou W and Restifo NP: T(H)17 cells in tumour immunity and immunotherapy. Nat Rev Immunol 10: 248-256, 2010.

38. Emri E, Egervari K, Varvolgyi T, Rozsa D, Miko E, Dezso B, Veres I, Mehes G, Emri G and Remenyik E: Correlation among metallothionein expression, intratumoural macrophage infiltration and the risk of metastasis in human cutaneous malignant melanoma. J Eur Acad Dermatol Venereol 27 e320-e327, 2013

39. Mantovani A and Locati M: Tumor-associated macrophages as a paradigm of macrophage plasticity, diversity, and polarization: Lessons and open questions. Arterioscler Thromb Vasc Biol 33: 1478-1483, 2013.

40. Folkman J: Angiogenesis. Annu Rev Med 57: 1-18, 2006.

41. Rolny C, Mazzone M, Tugues S, Laoui D, Johansson I, Coulon C, Squadrito ML, Segura I, Li X, Knevels E, et al: HRG inhibits tumor growth and metastasis by inducing macrophage polarization and vessel normalization through downregulation of PlGF. Cancer Cell 19: 31-44, 2011.

42. Luo Y, Zhou H, Krueger J, Kaplan C, Lee SH, Dolman C, Markowitz D, Wu W, Liu C, Reisfeld RA and Xiang R: Targeting tumor-associated macrophages as a novel strategy against breast cancer. J Clin Invest 116: 2132-2141, 2006.

43. Stockmann C, Doedens A, Weidemann A, Zhang N, Takeda N, Greenberg JI, Cheresh DA and Johnson RS: Deletion of vascular endothelial growth factor in myeloid cells accelerates tumorigenesis. Nature 456: 814-818, 2008.

44. Fantin A, Vieira JM, Gestri G, Denti L, Schwarz Q, Prykhozhij S, Peri F, Wilson SW and Ruhrberg C: Tissue macrophages act as cellular chaperones for vascular anastomosis downstream of VEGF-mediated endothelial tip cell induction. Blood 116: 829-840, 2010.

45. Chen C, Sun SR, Gong YP, Qi CB, Peng CW, Yang XQ, Liu SP, Peng J, Zhu S, Hu MB, et al: Quantum dots-based molecular classification of breast cancer by quantitative spectroanalysis of hormone receptors and HER2. Biomaterials 32: 7592-7599, 2011.

46. Peng CW, Tian Q, Yang GF, Fang M, Zhang ZL, Peng J, Li Y and Pang DW: Quantum-dots based simultaneous detection of multiple biomarkers of tumor stromal features to predict clinical outcomes in gastric cancer. Biomaterials 33: 5742-5752, 2012. 